\title{
Expression of the genes encoding kinin receptors are increased in human carotid atherosclerotic plaques
}

\author{
YAPEI GUO ${ }^{*}$, TIANTIAN LIU ${ }^{2 *}$, XUEYUAN LI ${ }^{3}$, MIN ZHANG $^{1}$, LEI SHI $^{1}$ and HENGFANG LIU ${ }^{1}$ \\ ${ }^{1}$ Department of Neurology, Fifth Affiliated Hospital, Zhengzhou University, Zhengzhou, Henan 450052; \\ ${ }^{2}$ Department of Neurology, Xiangya Hospital, Central South University, Changsha, Hunan 410008; \\ ${ }^{3}$ Department of Neurosurgery, Peking Union Medical College Hospital, Chinese Academy \\ of Medical Sciences and Peking Union Medical College, Beijing 100730, P.R. China
}

Received December 24, 2014; Accepted January 20, 2015

DOI: $10.3892 /$ br.2015.421

\begin{abstract}
There is increasing evidence showing that inflammation occurs in atherosclerosis and contributes to the formation of atherosclerotic plaques. As important inflammatory peptides, kinins are increased in inflammation, eliciting vasodilation, increasing vascular permeability and recruiting inflammatory cells to the injury sites by activating specific receptors, $\mathrm{B} 1$ and $\mathrm{B} 2$. The two receptors have been reported to increase in inflammation, but their expressions remain to be defined in human carotid atherosclerotic plaques (CAP). In order to assess the gene expression of kinin receptors in human CAP, 47 CAP specimens were collected from patients undergoing endarterectomy and classified into stable and unstable plaque groups, respectively, with 10 mesenteric arteries used as controls. Total mRNA of B1R and B2R was extracted from CAPs and their levels were determined using reverse transcription-polymerase chain reaction. The expression of $B 1 R$ and $B 2 R$ mRNA was significantly upregulated in human CAPs compared to the control arteries. In the unstable plaques, the ratios of $B I R$ to the $\beta$-actin mRNA level were significantly increased relative to the stable plaques. However, no notable differences were observed in the ratios of $B 2 R$ to $\beta$-actin in mRNA expression between the stable and unstable plaques. The present study suggests that kinin-mediated inflammation involves the formation of atherosclerotic plaque and $B 1 R$ plays an important role in plaque instability, indicating that kinin receptors can be used as potential targets for future therapeutic interventions.
\end{abstract}

Correspondence to: Professor Hengfang Liu, Department of Neurology, Fifth Affiliated Hospital, Zhengzhou University, 3 Kang-Fu Street, Zhengzhou, Henan 450052, P.R. China

E-mail: hengfangliu@yahoo.com.cn

*Contributed equally

Key words: atherosclerosis, carotid atherosclerotic plaques, kinin receptor

\section{Introduction}

Stroke, including local and widespread infarction, is a common cause of mortality and disability in patients with brain ischemia in developed and developing countries (1). The major cause of stroke is cerebral artery occlusion due to local thrombosis ensuing following the rupture of carotid atherosclerotic plaques (CAP) $(2,3)$. Among the various causes that influence the destabilization and rupture of atherosclerotic plaques, inflammatory reactions take an important place (4). The mRNA expression of numerous genes involved in inflammatory reactions is also altered in atherosclerotic plaques $(5,6)$.

The kinins, as a family of inflammatory peptides, are involved in a variety of physical processes, including vasodilation and vascular permeability (7). During inflammatory conditions, kinins induced vasodilation and pain, increased vascular permeability and recruited inflammatory cells to the sites of injury by activating specific receptors, B1 and B2 (8). $\mathrm{B} 1$ receptors $(\mathrm{B} 1 \mathrm{R})$ are weakly expressed under normal physiological circumstances, but their expression could be increased markedly by inflammation and injury (9). By contrast, B2 receptors (B2R) are expressed consecutively in healthy and pathological tissues and could also be enhanced in the inflammatory processes (8). Activated kinin receptors increased the production of endothelial nitric oxide (NO) and prostaglandins by enabling NO synthase and phospholipase A2 in endothelial cells. NO and prostaglandins act on the smooth muscle, leading to vasodilation, increased permeability and pain. In addition, kinins also interacted directly with its receptors located in vascular smooth muscle cells, resulting in the activation of several enzymes, such as mitogen-activated protein kinases and protein kinase $\mathrm{C}$, accompanied with the generation of reactive oxygen species, cellular migration and production of extracellular matrix proteins (10).

Previously, a number of studies have shown the increased expression of $\mathrm{B} 1 \mathrm{R}$ and $\mathrm{B} 2 \mathrm{R}$ in atherosclerotic vessels and peripheral blood cells of patients with atherosclerosis $(11,12)$. Particularly, in the cardiovascular systems, kinins have been found to be involved in the pathogenesis of heart failure (13). Notably, whereas kinins promoted angiogenesis, they also 
inhibited post-angioplasty restenosis (14). However, despite the extensive studies focused on the kinin-mediated inflammation in various pathologies, little is known regarding their performance in the formation and destabilization of human CAP.

In the present study, the expression of the genes encoding kinin receptors in human CAPs were assessed and compared to arteries obtained from normal controls, to improve the understanding of the role of kinin-mediated inflammation in the formation of human CAP, and therefore develop new preventive strategies. In addition, the plaques were classified into two groups based on the presence of surface ruptures, so as to further assess the performance of the kinin receptors in the destabilization of atherosclerotic plaques.

\section{Patients and methods}

Patients. Carotid plaque specimens from 47 patients undergoing carotid endarterectomy for symptomatic (transient ischemic attacks and minor strokes) or asymptomatic stenosis $>70 \%$, as confirmed by magnetic resonance angiography (MRA) or conventional arteriography, were collected. Each patient was subjected to a detailed history assessment and a physical examination, including routine blood biochemistry tests, electrocardiography, chest radiography, ultrasound cardiograph, carotid contrast-enhanced magnetic resonance imaging, computed tomography, magnetic resonance imaging and MRA of the brain. The vascular risk factors, as well as previous antihypertensive, statin and antiplatelet treatments, were also recorded (Table I). A total of 10 inferior mesenteric arteries dissected from colectomy specimens following elective surgery were used as controls. Each control patient was assessed to exclude symptomatic atherosclerotic disease by history and examination.

The study was approved by the Medical Ethics Committee of The Fifth Affiliated Hospital of Zhengzhou University (Zhengzhou, China). Written informed consent was obtained from each participant.

Specimens. The endarterectomy specimens included the atheromatous plaque, the adjacent intima and the medial layers. The control arteries were full-thickness and consisted of the adventitial layer. Plaques and control arteries were frozen immediately in liquid nitrogen and stored at $-80^{\circ} \mathrm{C}$ until further processing. Based on the presence of surface ruptures on plaques, the specimens were assigned into two groups: The stable plaque group, which contained a diffuse intimal thickening, a small eccentric plaque or a necrotic core surrounded by fibrous tissue without surface ruptures; and the unstable plaque group, which contained complex plaques with a possible surface defect, hemorrhage or thrombus.

RNA extraction. Total RNA was extracted from freshly isolated pulverized frozen tissue $(\sim 25 \mathrm{mg})$ with TRIzol ${ }^{\circledR}$ reagent (Invitrogen Life Technologies, Carlsbad, CA, USA) following the manufacturer's instructions and was solubilized in RNAase-free water. All the extracts were treated with DNase to avoid contamination from genomic DNA. Final concentration and purity were qualitatively evaluated by electrophoresis in $1 \%$ agarose gel stained with ethidium bromide and quantified
Table I. Demographic and clinical data of patients and controls.

\begin{tabular}{lccc}
\hline Variables & Patients & Controls & P-value \\
\hline Number & 47 & 10 & \\
Age, years & $56.1 \pm 7.3$ & $55.9 \pm 10.1$ & NS \\
Gender, male/female & $33 / 14$ & $7 / 3$ & NS \\
Smoking & 26 & 4 & NS \\
Diabetes & 12 & 1 & NS \\
Hypertension & 30 & 3 & NS \\
Coronary artery disease & 14 & 0 & NS \\
Family of IHD & 22 & 2 & NS \\
Aspirin & 19 & 1 & NS \\
Clopidogrel & 2 & 0 & NS \\
Warfarin & 4 & 0 & NS \\
Statins & 14 & 0 & NS \\
\hline
\end{tabular}

IHD, ischemic heart disease; NS, not significant.

spectrophotometrically (Nanodrop DA-2000; Thermo Fisher Scientific, Wilmington, DE, USA).

Primers. The primers and probes for amplification of $B 1 R$ and $B 2 R$ were determined using the Primer Premier 6.0 computer program (Premier Biosoft, Palo Alto, CA, USA) and their sequence homologies were checked using the GenBank database (www.ncbi.nlm.nih.gov/genbank).

The primer sequences used were as follows: i) BlR forward, 5'-ACGATTCTCCCACCTCA-3'; and reverse, 5'-AGCCCA AGACAAACACCA-3'; and ii) $B 2 R$ forward, 5'-CTACCC AGCCCTTGAAAGAT-3'; and reverse, 5'-GAAGACGCT GAGGACAAAGAT-3'.

The $\beta$-actin gene was used as the endogenous control and amplified simultaneously with the target gene. The primer sequences used for $\beta$-actin were as follows: Forward, 5'-AAG GAAGGCTGGAAGAGTGC-3'; and reverse, 5'-CTGGGA CGACATGGAGAAAA-3'.

Reverse transcription-polymerase chain reaction (RT-PCR). The mRNA level was determined by RT-PCR. The first-strand cDNA was synthesized from $2 \mu \mathrm{g}$ total RNA with PrimeScript Reverse Transcriptase (Takara Bio, Inc., Shiga, Japan). PCR was carried out by incubating each cDNA sample with primers (0.5 $\mu \mathrm{M}$ each), Blend Taq ${ }^{\circledR}$ polymerase (1.25 U; Takara Bio, Inc.) and a deoxynucleotide. The cycling conditions were as follows: $\mathrm{RT}, 42^{\circ} \mathrm{C}$ for $60 \mathrm{~min}$; polymerase activation, $70^{\circ} \mathrm{C}$ for $15 \mathrm{~min}$; PCR, 35 cycles at $94^{\circ} \mathrm{C}$ for $3 \mathrm{~min}$ and $94^{\circ} \mathrm{C}$ for $30 \mathrm{sec}$; and final elongation, $72^{\circ} \mathrm{C}$ for $40 \mathrm{sec}$. Reactions were performed in triplicate and in a total volume of $25 \mu \mathrm{l}$. PCR products were electrophoresed in $1 \%$ agarose gels. Bands were stained with ethidium bromide (Sigma, St. Louis, MO, USA) and detected with an LAS-3000 mini CCD camera (FUJIFILM Investment Co., Ltd., Shanghai, China). The Eagle Eye II analyzer (Strategene, La Jolla, CA, USA) was used to determine the optical density (OD) of the target and reference mRNA. The mRNA level was recorded as the relative value of the OD of the target gene to that of $\beta$-actin. 
A

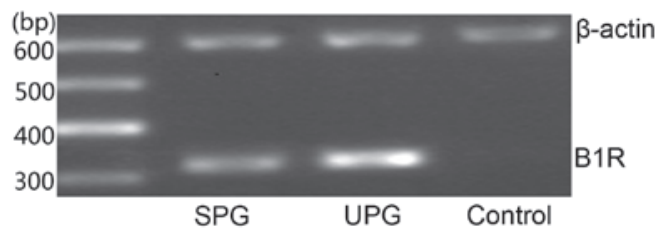

B

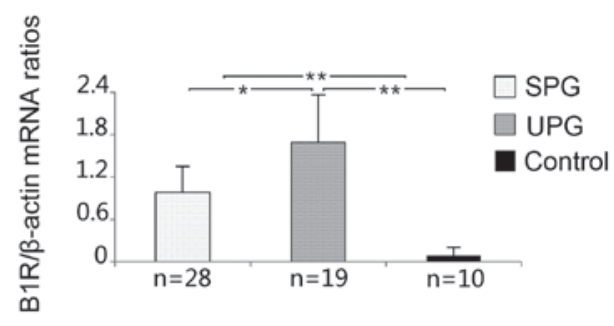

Figure 1. (A) Polymerase chain reaction results for $\beta$-actin and $B 1 R$ in the stable plaque group (SPG), unstable plaque group (UPG) and control group. (B) Differences in the relative ratios of $B 1 R$ to $\beta$-actin in the mRNA expressions across the different groups. ${ }^{*} \mathrm{P}<0.05 ;{ }^{* *} \mathrm{P}<0.05$.

Statistical analysis. Results are presented as mean \pm standard error of the mean. Statistical analysis was performed with SPSS 17.0 (SPSS, Inc., Chicago, IL, USA). Gender differences between patients and control groups were assessed using the $\chi^{2}$ test. Comparisons in the $B 1 R$ and $B 2 R$ mRNA levels across different groups of patients and controls were performed using analysis of variance. $\mathrm{P}<0.05$ was considered to indicate a statistically significant difference.

\section{Results}

The demographic and clinical data of the patients and controls are shown in the Table. I. There was no significant difference between patients and controls in gender, symptomatic carotid disease, individual or combined risk factors, treatment or coexistence of other vascular disease (Table. I).

One specimen was collected from each patient and 47 plaques were obtained in total. These specimens were classified into two groups respectively, based on the presence of surface ruptures on plaques: i) The stable plaque group with no defects on the surface $(n=28)$; and ii) the unstable plaque group with surface defects or ruptures $(n=19)$.

Increased expression of the genes encoding $B 1 R$ was observed in the stable and unstable plaque groups relative to the control group $(0.98 \pm 0.37$ and $1.69 \pm 0.65$ vs. $0.07 \pm 0.06)$. The $B I R$ mRNA expression was significantly increased in the plaque groups as compared to the control group (Fig. 1). When comparing the two plaque groups, the relative ratios of $B I R$ to $\beta$-actin in the mRNA level in the unstable plaque group were notably higher than that in the stable plaque group (Fig. 1).

The expression of the genes encoding $B 2 R$ were also increased in the stable and unstable plaque groups as compared to that in the control group $(0.98 \pm 0.43$ and $1.16 \pm 0.52$ vs. $0.48 \pm 0.16$ ). The differences in the relative ratios of $B 2 R$ to $\beta$-actin in the mRNA expression between the plaque groups and control group were significant (Fig. 2). When the two plaque groups were compared, no notable difference was identified in the relative ratios of $B 2 R$ to $\beta$-actin in the mRNA expression.
A

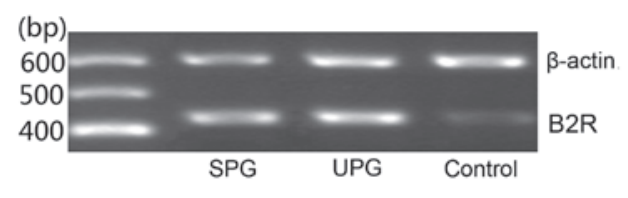

B

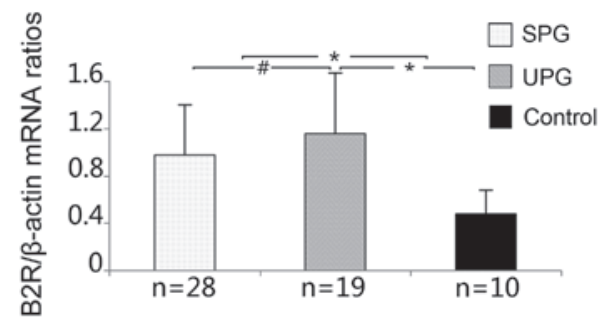

Figure 2. (A) Polymerase chain reaction results for $\beta$-actin and $B 2 R$ in the stable plaque group (SPG), unstable plaque group (UPG) and control group. (B) Differences in the relative ratios of $B 2 R$ to $\beta$-actin in the mRNA expressions across the different groups. ${ }^{\#} \mathrm{P}<0.05 ;{ }^{*} \mathrm{P}<0.05$.

\section{Discussion}

In the present study, the $B 1 R$ and $B 2 R$ mRNA expression in human CAPs was significantly unregulated as compared to control arteries, suggesting that kinin-mediated inflammation contributes to the formation of atherosclerotic plaques, which is in accordance with previous findings showing that kinin receptors were increased in the cardiovascular and peripheral vessel atherosclerosis $(4,15,16)$. When the plaques were classified into two groups based on the presence of surface ruptures, the relative ratios of $B 1 R$ to $\beta$-actin in the mRNA expression in the unstable plaque group were higher than that in the stable plaque group. However, no significant differences were observed in the relative ratios of $B 2 R$ to $\beta$-actin in the mRNA levels between the stable and unstable plaque groups. These findings may indicate that the $B 1 R$ gene expression contributes to the destabilization or rupture of human CAP. To the best of our knowledge, similar studies focusing on the role of kinin-mediated inflammation in the destabilization of human CAP have not been reported previously.

Traditionally, it is believed that the formation of atherosclerotic plaques simply reflects the deposition of lipids within the vessel wall of medium-sized and large arteries $(15,16)$. Currently, it is recognized that inflammation, including focal and systematic inflammation, are important players in the atherosclerotic processes (15). As important inflammation members, the kinin systems are also involved in the regulation of vasomotor, endogenous fibrinolysis in humans and their biological effects are mediated by two $\mathrm{G}$ protein-coupled receptors, B1 and B2 $(7,15)$. As stated, B2R is constitutively expressed in the majority of tissues under normal conditions. In the cardiovascular system, B2R is mainly located on the surface of endothelial cells and inflammatory cells, including monocytes, which can promote the vasodilation and fibrinolysis by acting through $\mathrm{NO}$ and prostaglandin (8). In contrast to B2R, B1R is hardly expressed under physical conditions, but its expression could be markedly increased under inflammatory conditions, which triggers the release of numerous inflammatory mediators, including tumor necrosis factors- $\alpha$ and interleukins $(17,18)$. The activated monocytes 
have a higher expression of B1R, but the B2R activity tends to decrease (19). Dabek et al (4) reported a higher increase in the $B 1 R$ gene expression in the mononuclear cells from patients with atherosclerosis. The dysfunction of endothelial cells and monocyte infiltration are two important contributors in the formation of atherosclerotic plaques, particularly for those with surface ruptures, which results in the accumulation of monocytes and dysfunctional endothelial cells accompanied with the subsequent upregulation of $B 1 R$ and $B 2 R$ (16). Consistent with prior findings, our present study showed the notable increased expression of $B 1 R$ and $B 2 R$ mRNA in human CAPs as compared to controls, providing evidence for the hypothesis that kinin-mediated inflammation contributes to the formation of human CAP.

Previous studies have shown that the instability of atherosclerotic plaques in the cycling system is in part determined by focal factors, but systemic factors such as inflammation and infections may also be extremely important components (20). In particular, the acute ischemic events have been reported to be more closely associated with the morphological features rather than the number of plaques or degree of stenosis $(20,21)$. Pathological studies of endarterectomy specimens demonstrated the plaque ulceration and rupture as the morphological features of previously symptomatic stenosis compared to asymptomatic plaques (21-23). Further comparison between symptomatic and asymptomatic endarterectomy specimens showed that the plaque areas covered by inflammatory cells were notably larger in symptomatic CAPs than asymptomatic ones, suggesting the involvement of inflammation in the plaque destabilization $(16,24)$. In the present study, the $B 1 R$ gene expression was significantly upregulated in the unstable plaques relative to stable ones, indicating that kinin-mediated inflammation plays an important role in the instability of human CAPs via $B 1 R$. Such a finding also provides useful information for developing novel therapies targeted at stabilizing the atherosclerotic plaques based on the systematic inflammation.

As mentioned above, kinin receptors are widely expressed in the endothelial cells and monocytes in the peripheral blood in the cycling systems. Apart from their role in the formation of human atherosclerotic plaques (19), the kinin receptors also play an important role in the instability of the plaques in the cardiovascular systems and carotid artery $(15,25,26)$, making them ideal targets for preventing and treating systematic atherosclerosis. Furthermore, it has been demonstrated on experimental models that the blockage of $\mathrm{B} 1 \mathrm{R}$ may reduce the systematic inflammatory response and therefore prevent the formation of atherosclerotic plaques (27). The impacts of kinin-mediated inflammation on the development of atherosclerotic plaques and their destabilization may open new avenues for future treatments.

However, the inflammation reactions mediated by kinins in the formation of human CAP are complex processes that involve numerous molecules and multiple signal pathways. The present study showed the involvement of the kinin-mediated inflammation in the formation and destabilization of human CAP on the mRNA level, but did not determine the subsequent protein expressions following translation as they are beyond the focus of the present study. However, the subsequent signaling pathways following receptor expression may also be quite complex processes, which require further investigation $(10,11)$.
In addition, the blockage of kinin receptor expression may prevent the formation of CAPs, a hypothesis that should be tested in future studies.

In conclusion, the present study demonstrated that the patients with CAP experienced notable alterations in the kinin receptor expression at the gene level. The significant upregulation of $B 1 R$ and $B 2 R$ mRNA expression in human CAPs revealed that the kinin-mediated inflammation played an important role in the atherosclerotic processes and also disclosed them as novel therapeutic targets for preventing the formation of atherosclerotic plaques. The unstable atherosclerotic plaques exhibited a higher ratio of $B I R$ to $\beta$-actin in mRNA levels, suggesting the increased $B 1 R$ mRNA as a potential target for reducing the destabilization of plaques. Future studies are required to explore the precise mechanism of inflammation causing the development and destabilization of atherosclerotic plaques and develop novel specific therapeutics targeted at such a mechanism.

\section{Acknowledgements}

The present study was supported by grants from the Zhengzhou Committee of Science and Technology (no. 083SGY2612-9).

\section{References}

1. Thorvaldsen P, Kuulasmaa K, Rajakangas AM, Rastenyte D, Sarti C and Wilhelmsen L: Stroke trends in the WHO MONICA project. Stroke 28: 500-506, 1997.

2. Lammie GA, Sandercock PA and Dennis MS: Recently occluded intracranial and extracranial carotid arteries. Relevance of the unstable atherosclerotic plaque. Stroke 30: 1319-1325, 1999.

3. Hankey GJ: Potential new risk factors for ischemic stroke: What is their potential. Stroke 37: 2181-2188, 2006.

4. Dabek J, Kulach A, Smolka G, Wilczok T, Scieszka J and Gasior Z: Expression of genes encoding kinin receptors in peripheral blood mononuclear cells from patients with acute coronary syndromes. Intern Med J 38: 892-896, 2008.

5. Rothwell PM, Villagra R, Gibson R, Donders RC and Warlow CP: Evidence of a chronic systemic cause of instability of atherosclerotic plaques. Lancet 355: 19-24, 2000.

6. Liu HF, Cui KF, Wang JP, Zhang M, Guo YP, Li XY and Jiang C: Significance of ABCA1 in human carotid atherosclerotic plaques. Exp Ther Med 4: 297-302, 2012.

7. Moreau ME, Garbacki N, Molinaro G, Brown NJ, Marceau F and Adam A: The kallikrein-kinin system: Current and future pharmacological targets. J Pharmacol Sci 99: 6-38, 2005.

8. Leeb-Lundberg LM, Marceau F, Muller-Esterl W, Pettibone DJ and Zuraw BL: International union of pharmacology. XLV. Classification of the kinin receptor family: From molecular mechanisms to pathophysiological consequences. Pharmacol Rev 57: 27-77, 2005.

9. Marin-Castano ME, Schanstra JP, Neau E, Praddaude F, Pecher C, Ader JL, Girolami JP and Bascands JL: Induction of functional bradykinin $\mathrm{b}(1)$-receptors in normotensive rats and mice under chronic Angiotensin-converting enzyme inhibitor treatment. Circulation 105: 627-632, 2002.

10. Rodriguez JA, De la Cerda P, Collyer E, Decap V, Vio CP and Velarde V: Cyclooxygenase-2 induction by bradykinin in aortic vascular smooth muscle cells. Am J Physiol Heart Circ Physiol 290: H30-H36, 2006.

11. Calixto JB, Medeiros R, Fernandes ES, Ferreira J, Cabrini DA and Campos MM: Kinin B1 receptors: Key G-protein-coupled receptors and their role in inflammatory and painful processes. Br J Pharmacol 143: 803-818, 2004.

12. Tschöpe $C$, Heringer-Walther $S$ and Walther T: Regulation of the kinin receptors after induction of myocardial infarction: A mini-review. Braz J Med Biol Res 33: 701-708, 2000.

13. Tschöpe C, Heringer-Walther S, Koch M, Spillmann F, Wendorf M, Hauke D, Bader M, Schultheiss HP and Walther T: Myocardial bradykinin B2-receptor expression at different time points after induction of myocardial infarction. J Hypertens 18: 223-228, 2000 
14. Agata J, Miao RQ, Yayama K, Chao L and Chao J: Bradykinin $\mathrm{B}(1)$ receptor mediates inhibition of neointima formation in rat artery after balloon angioplasty. Hypertension 36: 364-370, 2000

15. Cruden NL, Lang NN, MacGillivray TJ, Uren NG, Fox KA and Newby DE: Vasomotor and fibrinolytic responses to kinin receptor agonists in the atherosclerotic human lower limb. Heart Vessels 27: 179-185, 2012.

16. Stoll $\mathrm{G}$ and Bendszus M: Inflammation and atherosclerosis: Novel insights into plaque formation and destabilization. Stroke 37: 1923-1932, 2006.

17. Bockmann S and Paegelow I: Kinins and kinin receptors: Importance for the activation of leukocytes. J Leukoc Biol 68 : 587-592, 2000

18. McLean PG, Perretti M and Ahluwalia A: Kinin B(1) receptors and the cardiovascular system: Regulation of expression and function. Cardiovasc Res 48: 194-210, 2000.

19. Raidoo DM, Ramsaroop R, Naidoo S, Muller-Esterl W and Bhoola KD: Kinin receptors in human vascular tissue: Their role in atheromatous disease. Immunopharmacology 36: 153-160, 1997.

20. Rossi A, Franceschini L, Fusaro M, et al: Carotid atherosclerotic plaque instability in patients with acute myocardial infarction. Int J Cardiol 111: 263-266, 2006.

21. Spagnoli LG, Mauriello A, Sangiorgi G, Fratoni S, Bonanno E Schwartz RS, Piepgras DG, Pistolese R, Ippoliti A and Holmes DR Jr: Extracranial thrombotically active carotid plaque as a risk factor for ischemic stroke. JAMA 292: 1845-1852, 2004
22. Nighoghossian N, Derex L and Douek P: The vulnerable carotid artery plaque: Current imaging methods and new perspectives. Stroke 36: 2764-2772, 2005.

23. Cipollone F, Prontera C, Pini B, et al: Overexpression of functionally coupled cyclooxygenase- 2 and prostaglandin $\mathrm{E}$ synthase in symptomatic atherosclerotic plaques as a basis of prostaglandin E(2)-dependent plaque instability. Circulation 104: 921-927, 2001

24. Nadareishvili ZG, Koziol DE, Szekely B, Ruetzler C, LaBiche R, McCarron R, DeGraba TJ and Jander S: Increased CD8(+) T cells associated with Chlamydia pneumoniae in symptomatic carotid plaque. Stroke 32: 1966-1972, 2001.

25. Lang NN, Cruden NL, Tse GH, Bloomfield P, Ludlam CA, Fox KA and Newby DE: Vascular B1 kinin receptors in patients with congestive heart failure. J Cardiovasc Pharmacol 52: 438-444, 2008

26. Newby DE, McLeod AL, Uren NG, Flint L, Ludlam CA, Webb DJ, Fox KA and Boon NA: Impaired coronary tissue plasminogen activator release is associated with coronary atherosclerosis and cigarette smoking: Direct link between endothelial dysfunction and atherothrombosis. Circulation 103: 1936-1941, 2001.

27. deBlois D and Horlick RA: Endotoxin sensitization to kinin B(1) receptor agonist in a non-human primate model: Haemodynamic and pro-inflammatory effects. Br J Pharmacol 132: 327-335, 2001. 